\section{T-CELL SIGNALLING}

\section{DRAK2 puts the brakes on T-cell responses}

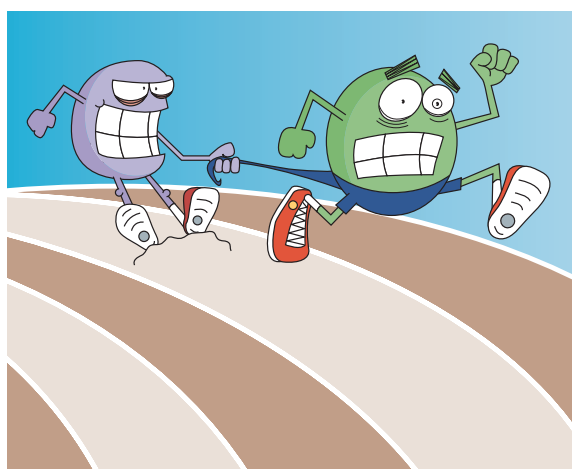

Death-associated protein-kinase-related 2 (DRAK2) is expressed specifically by lymphocytes and is a member of the death-associated protein (DAP)-like family of serine/threonine kinases, which induce apoptosis when overexpressed by cell lines. However, as reported recently in Immunity, mice that are deficient in
DRAK2 do not show defects in apoptosis; instead, T cells from these mice are hypersensitive to stimulation through the $\mathrm{T}$-cell receptor (TCR).

Apoptosis has a crucial role in many aspects of immune development and immune function, including negative selection of self-reactive thymocytes and clonal contraction of the expanded T-cell pool after antigenic stimulation. So, because DRAK2 is expressed exclusively in lymphoid tissues and is a member of the DAP-like family of kinases that are known to promote apoptosis, McGargill et al. sought to investigate whether DRAK2 regulates lymphoidcell apoptosis. DRAK2 was shown to be expressed at high levels by thymocytes (with expression increasing throughout development) and by mature T cells and B cells but not by other immune cells. Surprisingly, DRAK2deficient mice showed no defect in negative selection. However, double positive (DP) thymocytes from these mice expressed higher levels of CD5 and CD69 than wild-type DP thymocytes, indicating that DRAK2-deficient thymocytes receive a TCR signal of greater intensity. Consistent with this observation, DRAK2-deficient T cells proliferated more and produced more cytokine in response to TCR stimulation than wild-type T cells. Furthermore, whereas crosslinking of both CD3 and CD28 is required to induce purified wild-type T cells to proliferate, purified DRAK2-deficient $T$ cells proliferated in response to stimulation with CD3-specific antibody alone. Because the augmented proliferation of DRAK2-deficient $\mathrm{T}$ cells was not a result of increased cell survival after activation - that is, the defect was not in the cell-death pathways that are associated with activation - these results are consistent with a role for DRAK2 in the negative regulation of TCR-induced signals.

Analysis of the signalling pathways that are affected by DRAK2 showed that both calcium flux and phosphorylation of the signalling molecules cJUN and extracellular signal-regulated kinase 1 (ERK1) and/or ERK2 was increased following TCR stimulation of DRAK2-deficient $T$ cells. By contrast, no difference in the phosphorylation of other signalling molecules, including TCR- $\zeta$, LCK and p38, was observed, indicating that specific pathways downstream of the TCR are influenced by DRAK2.

Mice that are deficient in other negative regulators of T-cell activation, such as CBL-B (Casitas

\title{
No need for the thymus
}

The mechanism of $\mathrm{CD} 4^{+} \mathrm{T}$-cell loss in infection with HIV is a hotly debated issue and has frequently been suggested to reflect impairment of thymic function resulting from viral pathology. However, a recent report in the European Journal of Immunologyrules out this mechanism and provides the first direct evidence indicating that the thymus has limited influence on peripheral T-cell loss in HIV infection.

To assess the role of the thymus in peripheral T-cell homeostasis, Linqi Zhang and colleagues thymectomized juvenile rhesus macaques. Analysis of the peripheral T-cell pool for ten months following surgery indicated that thymectomized animals had a small but significant reduction in peripheral T-cell numbers compared with control animals that had sham surgery, with the fastest decline being in the $\mathrm{CD} 4^{+} \mathrm{T}$-cell population. In the thymectomized animals, there was a small increase in the percentage of proliferating $\mathrm{T}$ cells, which the authors suggested might compensate for the loss of thymus-derived T cells. In addition, to follow the influence of thymectomy on the number of recent thymic emigrants in the periphery, the authors also measured T-cell receptor (TCR) excision circles (TRECs) — episomal DNA by-products present in cells that have undergone TCR rearrangement. As expected, TREC numbers declined in the thymectomized animals, whereas shamoperated macaques had stable TREC numbers. From analysis of the TREC decay rates in thymectomized animals, the authors were able to estimate the proportion of the total T-cell population that is produced by the thymus per day. So, although the thymus is crucial for the initial establishment of the T-cell repertoire, its ongoing contribution to the mature T-cell pool is small: only $0.2-0.3 \%$ of the peripheral T-cell pool migrated from the thymus each day, which is consistent with the relatively small effects seen in the periphery of thymectomized animals.

The authors next assessed how lack of thymic output affected the outcome of infection with simian immunodeficiency virus (SIV). Contrary to expectations, SIV infection of thymectomized animals did not result in higher viral loads or faster disease progression compared with SIV-infected control animals. Moreover, comparable levels of $\mathrm{CD}^{+} \mathrm{T}$-cell loss were seen in both thymectomized and sham-operated SIVinfected animals. Comparison of $\mathrm{CD} 4^{+} \mathrm{T}$-cell loss in thymectomized animals before and after SIV infection revealed that the fastest decay occurred after infection, indicating that most $\mathrm{CD} 4^{+} \mathrm{T}$-cell loss in SIV infection is not explained by the loss of thymic output. A significant decline in TREC numbers was also seen following SIV infection in both thymectomized and sham-operated animals, although the rate of decline was greater in thymectomized animals. Given that the number of TRECs per cell is influenced by cell proliferation and cell death, the authors suggest that this increased rate of TREC decay is attributable to the higher rates of $\mathrm{CD}^{+}$ T-cell proliferation in thymectomized animals and not to the lack of thymic output.

So, given that thymic output seems to have a limited role in maintaining peripheral $\mathrm{T}$-cell numbers, SIV-mediated impairment of thymic function might not be an important contributing factor to T-cell depletion in SIV infection.

Lucy Bird

(D) References and links ORIGINAL RESEARCH PAPER Tuttleton Arron, S. et al Impact of thymectomy on the peripheral $\mathrm{T}$ cell pool in rhesus macaques before and after infection with simian immunodeficiency virus. Eur. J. Immunol. 35, 46-55 (2005). 
B-lineage lymphoma B) and CTLA4 (cytotoxic T-lymphocyte antigen 4), also have T cells that are hypersensitive to suboptimal TCR stimulation and are either equally susceptible or more susceptible to autoimmune disease than control animals. However, there were no signs of spontaneous autoimmunity in DRAK2-deficient mice. Furthermore, DRAK2-deficient animals were resistant to induced experimental autoimmune encephalomyelitis (EAE), and this correlated with a decrease in the number of cells infiltrating the central nervous system.

This study identifies a new pathway of negative regulation of T-cell activation, which is mediated by DRAK2. Understanding the surprising observation that DRAK2-deficient mice are resistant to EAE but respond normally to infection with lymphocytic choriomeningitis virus will require further study, but the authors suggest that DRAK2 could be a specific target for the treatment of autoimmune disease.

Karen Honey

(Q) References and links ORIGINAL RESEARCH PAPER McGargill, M. A., Wen, B. G. Walsh, C. M. \& Hedrick, S. M. A deficiency in Drak2 results in a $T$ cell hypersensitivity and an unexpected resistance to autoimmunity. Immunity 21, 781-791 (2004).

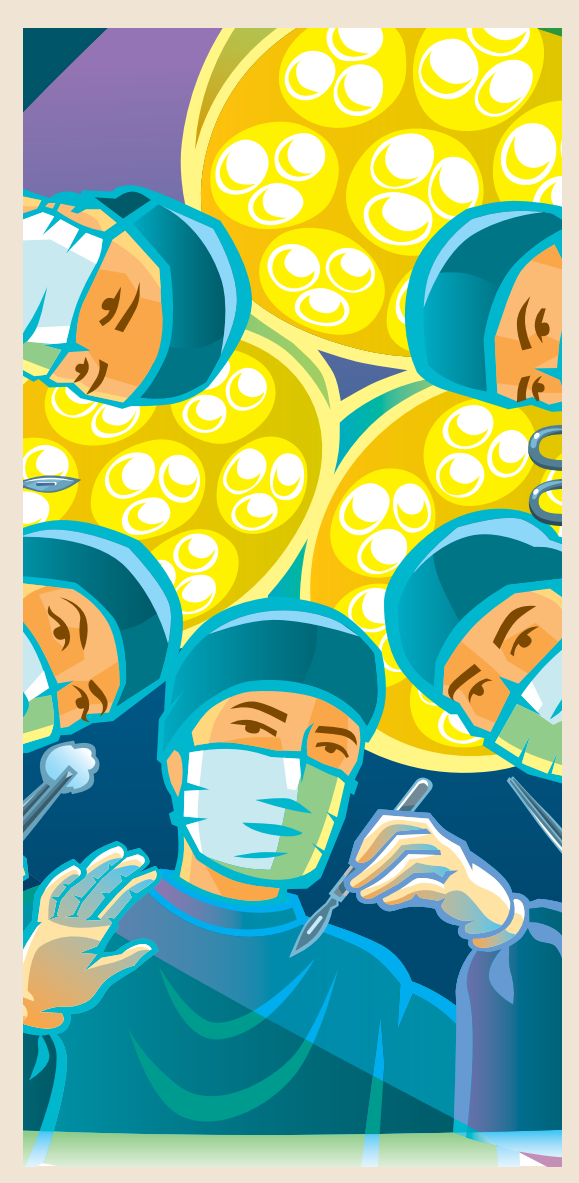

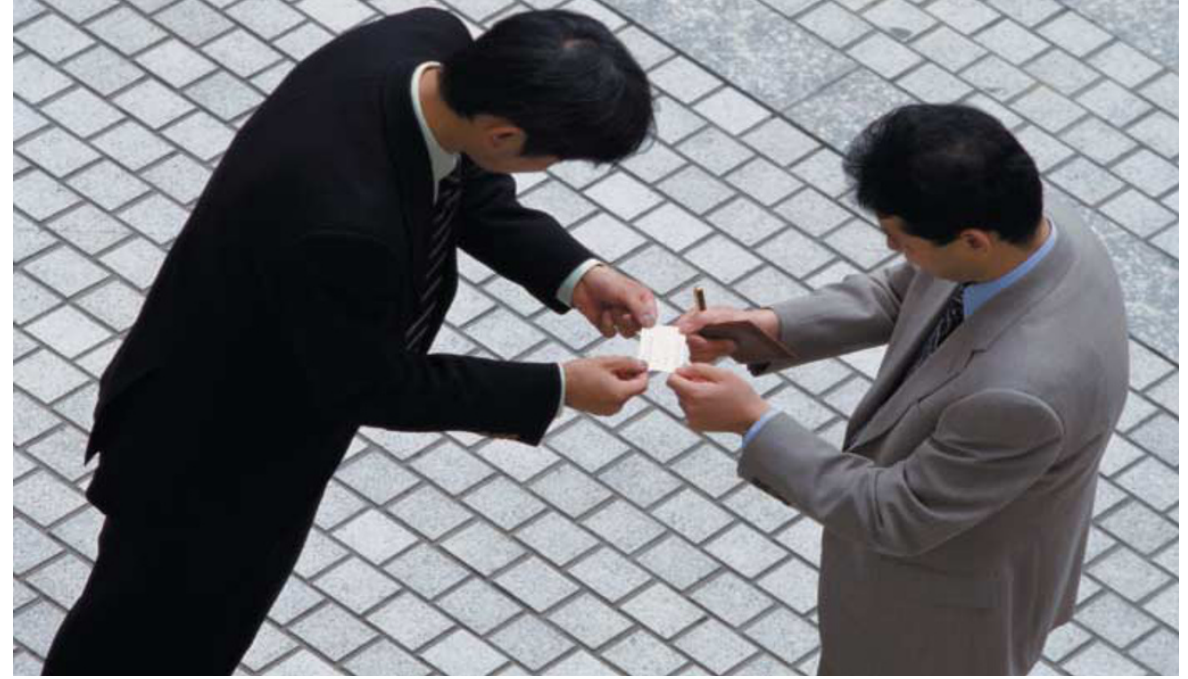

INFLAMMATION

\section{RIP2 leaves calling card for Crohn's disease}

The inflammatory bowel disease Crohn's disease is commonly thought to result from an inappropriate immune response to commensal bacteria in the gut. Although several genetic loci have been associated with disease susceptibility in humans, the link between these disease-associated mutations and intestinal inflammation is not entirely clear. This study further elucidates the involvement of one of the most common associated loci, CARD15, which encodes the cytoplasmic protein nucleotide-binding oligomerization domain 2 (NOD2).

After binding its ligand, muramyl dipeptide (MDP), which is derived from intracellular or phagocytosed bacteria, NOD2 is known to bind the protein kinase RIP2 (receptor-interacting protein 2), leading to activation of nuclear factor- $\kappa \mathrm{B}$ $(\mathrm{NF}-\mathrm{\kappa B})$ signalling through the inhibitor of NF- $\kappa \mathrm{B}$ (I $\kappa \mathrm{B}$ )-kinase complex (the IKK complex; which consists of IKK- $\alpha$, IKK- $\beta$ and NF- $\kappa \mathrm{B}$ essential modulator, NEMO).

Previous studies have led to the proposal that RIP2 has a scaffolding role, bringing NOD2 and IKK into close proximity, but Cantley and colleagues now show that RIP2 has a more active role. Co-expression of RIP2 with NEMO led to increased ubiquitylation of NEMO, which is associated with NF- $\kappa B$ activation. Use of a 'kinase-dead' RIP2 mutant showed that the protein-kinase activity of RIP2 is not required for this function. NOD2 was also shown to induce NEMO ubiquitylation, but only in the presence of the NOD2 agonist MDP. A lossof-function polymorphism in NOD2 that is associated with Crohn's disease reduced the level of NEMO ubiquitylation, and another
NOD2 mutation associated with more severe disease inhibited NEMO ubiquitylation entirely.

Next, they tested whether the NEMO ubiquitylation that is associated with MDPactivated NOD2 is due to the known binding of RIP2. Compared with wild-type NOD2, the disease-associated mutants bound significantly less RIP2. Furthermore, when the expression of RIP2 was inhibited by RNA interference, MDP-activated NOD2 failed to ubiquitylate NEMO. This indicates that RIP2 is required for the effects of NOD2 on the NF- $\kappa B$-signalling pathway and that the NOD2 mutants are associated with Crohn's disease as a result of their failure to bind RIP2 and activate the NF- $\kappa B$ pathway.

Although ubiquitylation of proteins is commonly associated with proteasomal degradation, addition of a proteasome inhibitor to cells producing RIP2 and NEMO did not alter the level of expression of NEMO, showing that NEMO ubiquitylation does not lead to degradation. Instead, NEMO ubiquitylation through the NOD2-RIP2 pathway increases NF- $\kappa$ B activity, as shown by a decrease in activity in the presence of a deubiquitinase. Failure to activate NF- $\kappa B$ signalling through this pathway provides a novel mechanism for the dysregulated inflammatory responses in Crohn's disease.

\footnotetext{
(9) References and links ORIGINAL RESEARCH PAPER Abbott, D. W., Wilkins, A Asara, J. M. \& Cantley, L. C. The Crohn's disease protein, NOD2, requires RIP2 in order to induce ubiquitinylation of a novel site on NEMO. Curr. Biol. 14, 2217-2227 (2004). FURTHER READING Inohara, N. \& Nuñez, G. NODs: intracellular proteins involved in inflammation and apoptosis. Nature Rev. Immunol. 3, 371-382 (2003) | Bouma, G. \& Strober, W. The immunological and genetic basis of inflammatory bowel disease. Nature Rev. Immunol. 3, 521-533 (2003).
} 